\title{
Congress takes holiday while Zika spreads and kills
}

Volume 4 Issue 2 - 2016

\section{Opinion}

When the USA citizenry first became aware of the Zika epidemics in South America and Africa, is was thought interesting in that many of the pregnant women who had contracted it delivered babies with microcephaly. But the worst was yet to come - and still is.

We have since learned that it is communicable (person to person) $;^{1}$ elicits a polio-like syndrome ${ }^{2}$ that can, like polio, result in neuromuscular disabilities (consider the cost to the SS disability program); ${ }^{3}$ and is deadly. ${ }^{4}$ There is no vaccine or pharmacological treatment for it.

In February of this year, President Obama requested 1.1billion dollars for the fight against Zika. Congress has left Washington for a seven-week summer vacation without definitive action on the matter. The administration has allocated $\$ 589$ million from existing funds to fight the outbreak - about $\$ 510$ million of that is from Ebola funding. The money is to go for development of a vaccine against and a pharmacological treatment of Zika.

There is a project now completing clinical studies that accomplished reduction of the viral load in overwhelming viremia. Aethlon Medical, Inc. has created a device that can remove massive amounts of virus, bacteria, and other pathogenic agents from patients' circulating blood. ${ }^{5}$ Use of Aethlon's Hemopurifier ${ }^{\circledR}$ is not claimed to cure any pathogenic blood invasion, but by reducing the massive amount of virus in the circulation, it can allow the previously overwhelmed immune system to recover so that it, in conjunction with a specific pharmacological agent, can then clear the body of the offending pathogen and, in effect, cure the viremia. This has been demonstrated in clinical studies ofw HIV and HCV for which there are effective drugs, if tolerated by the patient. In the one case involving Ebola, for which there is no effective drug, reduction in the viral load did enable the recovery of the immune system, apparently resulting in a cure.

Now, considering Zika, for which there is no effective pharmacological agent or vaccine, is it possible that the result could be as that for Ebola? Could capturing and removing millions of virus copies result in reawakening and recovery of the immune system and facilitate a cure of the disease? We cannot know unless the FDA approves an experimental study of infected humans as has been done in cases of HIV, HCV, and Ebola, remembering that in the first two, there are effective antiviral drugs that patients may or may not tolerate, while for Ebola, there is none.

It should be noted that Aethlon is working under a DARPA contract to develop and produce devices using the Hemopurifier ${ }^{\circledR}$ that can be operated on the battlefield by technicians to fight bacterial sepsis. Historically, sepsis has been the most common cause of death from battlefield injuries, more than actual battlefield injuries: '... infectious diseases have been responsible for the majority of deaths during war; however, numerous medical and military advances have reversed this trend, re-sulting in more deaths from battle than infectious diseases in the 20th century. In addition, there have been remarkable improvements decreasing the mortality rate from combat wounds during each major US conflict in the 20th century. ${ }^{6}$

\author{
Franklin David Nash \\ Center for Scientific Analysis of Policy, LLC, USA
}

Correspondence: Franklin David Nash, Principal, Center for Scientific Analysis of Policy, LLC, 7500 Hoover Road, Indianapolis, Tel 844.870.7870,Email docnash@scipolicy.org

Received: July 3I, 2016 | Published: August 04, 2016

Aethlon will be seeking approval and sufficient funding to enable the company to carry out a clinical trial against Zika, running parallel to others engaged in developing immunization and treatment options.

\section{Acknowledgments}

None.

\section{Conflicts of interest}

Author declares there are no conflicts of interest.

\section{Funding}

None.

\section{References}

1. https://images.search.yahoo.com/yhs/search; y $1 \mathrm{t}=\mathrm{A} 0 \mathrm{~L}$ E V r b $70 \mathrm{~J}$ B X p Z M A E U P X Q t .; y $1 \mathrm{u}=\mathrm{X} 3$ o D M T B y M j B 0 a G 5 z B G N v b G 8DYmYxBHBvcwMxBHZ0aW\%20Q\%20DBHN1YwNzYw--?p= Zika+Communicable\&fr=yhs-adk-adk_sbnt\&hspart=adk\&hsimp=yhsadk_sbnt

2. http://www.cdc.gov/zika/about/gbs-qa.html

3. As a trauma surgery resident in the my team handled fractures three days a week. This was not simply because we needed that much experience and training but because orthopedics were occupied doing surgery on post-polio patients to allow them to better use the upper and lower extremities. The cost was born from federal tax revenues and was enormous. 1960 .

4. http://news.sky.com/story/zika-linked-to-three-deaths-from-raredisorder-10157153

5. Nash FD. Reduction of Pathogenic Load in Overwhelming Viremia. Int J Complement Alt Med. 2016;3(6):00098.

6. Clinton K, Murray, Mary K, et al. History of Infections Associated With Combat-Related Injuries. J Trauma. 2008;64:S221-S231. 